\title{
Snoring, sleep disturbance, and behaviour in 4-5 year olds
}

\author{
N J Ali, D J Pitson, J R Stradling
}

\begin{abstract}
Parents of 996 children aged 4-5 years identified consecutively from the Oxford health visitor register were asked to complete a questionnaire about breathing disorders during sleep. A total of $782(78.5 \%)$ was returned. Ninety five $(12 \cdot 1 \%)$ children were reported to snore on most nights. Habitual snoring was significantly associated with daytime sleepiness, restless sleep, and hyperactivity.
\end{abstract}

The questionnaire responses were used to select two subgroups, one at high risk of a sleep and breathing disorder and a control group. These childiren (132 in total) were monitored at home with overnight video recording and oximetry, and had formal behavioural assessment using the Conners scale.

Seven (7/66) children from the high risk group and none from the control group had obvious sleep disturbance consequent on snoring and upper airway obstruction. Thus our estimate of the prevalence of sleep and breathing disorders in this age group is $7 / 996$ or $0 \cdot 7 \%$.

The high risk group had significantly higher nocturnal movement, oxygen saturation dip rates, and overnight pulse rates than the controls. Maternal but not paternal smoking was associated with the high risk group. Parents and teachers thought those in the high risk group were more hyperactive and inattentive than the controls, but only their parents thought them more aggressive.

Significant sleep and breathing disorders occur in about $0.7 \%$ of $4-5$ year olds. Children whose parents report snoring and sleep disturbance have objective evidence of sleep disruption and show more behaviour problems than controls.

(Arch Dis Child 1993; 68: 360-366)

Sleep and breathing disorders are increasingly recognised in children and are usually caused by a combination of adenotonsillar hypertrophy and decreased oropharyngeal dimensions. ${ }^{1}$ There has been a dramatic decrease in tonsillectomy rates in children up to 9 years of age from about $40 \%$ in the $1950 \mathrm{~s}^{2}$ to less than $1 \%$ by the same age in the years $1975-80 .^{3}$ This decreasing trend, and the often non-specific and poorly recognised symptoms associated with sleep and breathing disorders in children, ${ }^{+}$has led to the suggestion that the prevalence and consequences of these sleep related problems is increasing. ${ }^{5}$ The indications for adenotonsillectomy have also changed; one study found that obstructive sleep apnoea, which was not reported in 1978, accounted for $19 \%$ of the procedures in $1986 .{ }^{\circ}$

, accounted for $19 \%$ of
This study has therefore addressed questions about the prevalence and psychological impact of undiagnosed sleep and breathing disorders in children: first, through a questionnaire survey about snoring and sleep problems in 996 randomly selected children aged $4 \cdot 5-5$ years; and second, by evaluating with home sleep studies and formalised behavioural assessments a high risk subgroup and comparing these children with a low risk control subgroup, with the two groups selected from the initial survey.

\section{Subjects}

QUESTIONNAIRE SURVEY

Nine hundred and ninety six boys and girls born consecutively between 8 January and 22 September 1985 and living in Oxford were identified from the centralised health visitor register. The ages at the time of study ranged from $4 \cdot 5$ to $5 \cdot 5$ years.

\section{SLEEP STUDY AND BEHAVIOURAL ASSESSMENT} GROUP

On the basis of the answers to the initial questionnaire, two subgroups were selected for further study. Questions 1, 5, 6, 9, 10, and 11 (essentially about snoring and other symptoms of sleep and breathing disorders; see appendix) were given a weighting based on their ability to predict the presence of sleep and breathing disorders in a previous study from this department. ${ }^{7}$ The overall score was then used to rank the children in order of the likelihood of them having a sleep and breathing disorder. The 66 highest ranking children (henceforth the high risk group) and an equal number of sex matched children from the lowest rankings (henceforth the control group) were studied further. Their parents were contacted by letter and telephone and asked if they would allow their children to take part in the study. Of 73 approached in the high risk group $66(90 \%)$ agreed to the study, whereas we contacted 88 in the control group to obtain an equal number to study $(75 \%)$. Thus 132 children from the original target population of $996(13.3 \%)$ had sleep studies and psychological assessment.

\section{Methods}

QUESTIONNAIRE SURVEY

The postal questionnaire inquired about snoring and other symptoms of sleep and breathing disorders in children (see Appendix) and was a reduced version of that used in an earlier study from this department. 
SLEEP STUDY AND BEHAVIOURAL ASSESSMENT GROUPS

General health, enuresis, parental smoking, and occupation questionnaire

We inquired about the child's general health (particularly hearing problems), drugs taken, and whether they had regular enuresis. The socioeconomic group was determined from paternal occupation according to the Office of Population and Censuses and Surveys (OPCS) definitions, ${ }^{8}$ and parental smoking habits were also determined as these factors might be potentially confounding variables in relating snoring and behavioural assessment. ${ }^{9}$

\section{Overnight video recordings}

A low light video camera, infrared light source, microphone, and video recorder were set up in the child's bedroom to run between $10 \mathrm{pm}$ and $6 \mathrm{am}$. The recordings were processed automatically for body movements as a measure of sleep disturbance ${ }^{70}$ which was expressed as the percentage of time spent moving. All video recordings were reviewed blind before the estimation of the percentage of time spent moving to remove periods of longer wakefulness, thus ensuring that this index was a measure of the transient arousals characteristic of sleep and breathing disorders. Any snoring and obstructed breathing producing arousals was documented to classify the children as normal, snorers, or snorers/sleep apnoea with sleep disturbance.

\section{Oximetry}

The Ohmeda 3700 pulse oximeter with an eight hour internal memory was used to make overnight recordings." By asking the parents to record accurately the time the oximeter was switched off in the morning we were able to synchronise the video and oximeter recordings to within a minute or so. The oximetry data were unloaded into a BBC microcomputer and the average oxygen saturation and pulse rate calculated. The number of dips in arterial oxygen saturation $\left(\mathrm{SaO}_{2}\right)$ greater than $4 \%$ was also calculated and expressed as $>4 \%$ dips per hour $(>4 \%$ $\mathrm{SaO}_{2}$ dip rate) as described previously. ${ }^{12}$

\section{Conners behaviour rating scale}

The Conners scale was shortened by including only the three subscales (out of a normal five) that related to the behaviour patterns reported in association with sleep apnoea in children (that is, aggression, inattention, and hyperactivity). ${ }^{13}$ We used the subscales and scoring system as reported by Conners. ${ }^{13}$

\section{Protocol}

QUESTIONNAIRE SURVEY

The questionnaire, together with a letter that indicated our interest in children's sleep in general (not specifically in sleep and breathing disorders), was sent to the parents of the 996 children identified from the health visitor records. If no reply was received within three to four weeks one reminder was sent.
SLEEP STUDY AND BEHAVIOURAL ASSESSMENT GROUPS

The two subgroups (132 children) identified from the initial questionnaire answers were visited at home and their parents completed the general health and parental smoking and occupation questionnaire. The sleep study equipment was set up in the child's bedroom and collected the next day. If the child appeared to have a current upper respiratory tract infection, then the sleep study was deferred until they had recovered. At a later date (within three months) the parents were asked to complete the Conners behaviour scale themselves and to pass another copy onto the child's teacher for completion.

\section{Analysis}

All the data were entered into the Oxford University VAX computer and analysed using the SAS suite of statistical programs ${ }^{1+}$ or the General Linear Interactive Modelling (GLIM) statistical package ${ }^{15}$ as appropriate.

\section{QUESTIONNAIRE SURVEY}

The questionnaire responses were examined for associations between reported snoring and daytime sleepiness, hyperactivity, and restless sleep. The original four possible responses to the snoring question were retained and given an arbitrary score of $1=$ never, $2=$ rarely, $3=$ sometimes even without a cold, and $4=$ most nights, whereas those for daytime sleepiness, hyperactivity, and restless sleep were collapsed into two categories: never/rarely and sometimes/ often. Linear trends across the snoring categories in daytime sleepiness, hyperactivity, and restless sleep were tested for statistical significance using $\chi^{2}$ trend analysis. ${ }^{16}$ The relative risks and $95 \%$ confidence intervals were calculated for habitual snorers (that is, most nights) compared with non-snorers (that is, never) using the confidence interval analysis computer program. ${ }^{17}$

\section{SLEEP STUDY AND BEHAVIOURAL ASSESSMENT} GROUPS

Determination of the prevalence of sleep and breathing disorders

There are no accepted criteria for the diagnosis of sleep and breathing disorders in children because there is a continuum from normality to gross disease with uncertain correlations and consequences. We chose to use the 95 th centile established for a group of normal children (previously reported from this unit ${ }^{7}$ which utilised identical techniques) to define the upper limit of normality for nocturnal movement (percentage of time spent moving), $>4 \% \mathrm{SaO}_{2}$ dip rate, and mean overnight pulse rate. The reviews of the video and oximetry records of these children (who were above the 'normal' range for one or more of the measurements) were then used to determine whether a sleep and breathing disorder or another factor was the likely explanation for their abnormal result.

Comparison of high risk and control groups Overnight sleep monitoring. The two groups 
Table 1 Effect of snoring on prevalence of daytime sleepiness, hyperactivity, and restless sleep $(n=782)$. Relative risk compares the habitual snorers to the never snorers

\begin{tabular}{|c|c|c|c|c|c|c|c|}
\hline & \multicolumn{4}{|c|}{ Snoring category } & \multirow[b]{2}{*}{$\begin{array}{l}\chi^{2} \text { trend } \\
(p \text { value })\end{array}$} & \multirow[b]{2}{*}{$\begin{array}{l}\text { Relative } \\
\text { risk }\end{array}$} & \multirow{2}{*}{$\begin{array}{l}95 \% \\
\text { Confidence } \\
\text { interval }\end{array}$} \\
\hline & $\begin{array}{l}\text { Never } \\
(n=247)\end{array}$ & $\begin{array}{l}\text { Rarely } \\
(n=239)\end{array}$ & $\begin{array}{l}\text { Sometimes } \\
(n=201)\end{array}$ & $\begin{array}{l}\text { Most nights } \\
(n=95)\end{array}$ & & & \\
\hline $\begin{array}{l}\text { Daytime sleepiness } \\
(\%)\end{array}$ & 19 & 17 & 26 & 31 & $\begin{array}{l}3.88 \\
(p=0.048)\end{array}$ & $1 \cdot 18$ & 1.02 to 1.36 \\
\hline Hyperactivity (\%) & 19 & 23 & 31 & 52 & 31.9 & $1 \cdot 74$ & $1 \cdot 38$ to $2 \cdot 18$ \\
\hline Restless sleep (\%) & 30 & 36 & 50 & 64 & $\begin{array}{l}39.3 \\
(\mathrm{p}<0.0001)\end{array}$ & $2 \cdot 06$ & 1.53 to 2.76 \\
\hline
\end{tabular}

were compared with respect to the percentage of time spent moving, $>4 \% \mathrm{SaO}_{2}$ dip rate, and pulse rate using an unpaired $t$ test.

Social class and parental smoking. For the purposes of analysis the six socioeconomic categories defined by the OPCS were collapsed into two: manual - that is, groups IIIm, IV and V - and non-manual - that is, groups I, II, and IIInm. Single logistic regression was used to calculate the effect of social class and parental smoking on the odds ratio for being in the high risk group. The odds ratios were then recalculated in a logistic regression model after allowing for potentially confounding variables - for example, for social class we entered maternal and paternal smoking into the analysis first. ${ }^{15}$

Conners child behaviour scale. To produce two categories to be used as the dependant variable in logistic regression we used the 95th centile for the control group to define the upper limit of 'normality' in our study. Thus any children whose scores equalled or exceeded this value were defined for the purposes of analysis as 'abnormal'. As in the social class and parental smoking analysis single logistic regression was used to determine the odds ratio effect of being in the high risk versus the control group on the score for each of the three subscales. The odds ratios were then recalculated in a logistic regression model after controlling for gender and social class as potentially confounding variables.

\section{Results}

QUESTIONNAIRE SURVEY

Six hundred and seven $(607 / 996 ; 60.9 \%)$ completed questionnaires were returned initially and a further 175 after the reminder letter (total returned $78 \cdot 5 \%)$. Of these, $417 / 782(53 \cdot 3 \%)$ were boys. Thirty per cent of the parents reported their children as having problems related to sleeping sometimes or often, $22 \cdot 3 \%$

Table 2 Summary of results of sleep monitoring in the two study groups, showing measuremen in which thev exceeded the normal range and categorised by whether they had a sleep and breathing disorder $(S B D)$, snored, or had apparently normal sleep when the video recording was reviewed

\begin{tabular}{|c|c|c|c|c|c|}
\hline & \multicolumn{3}{|c|}{ High risk group } & \multicolumn{2}{|c|}{ Control group } \\
\hline & $S B D$ & Snorer & 'Normal' & Snorer & 'Normal' \\
\hline$>4 \% \mathrm{SaO}_{2}$ dip rate & 2 & + & 5 & - & - \\
\hline Nocturnal movement & 2 & - & $3^{\star}$ & - & $t t^{\star}$ \\
\hline $\begin{array}{l}\text { Pulse rate } \\
\text { Pats }\end{array}$ & $=$ & - & 1 & - & - \\
\hline$>4 \% \mathrm{SaO}_{2}$ dip rate and movement & 3 & - & - & - & $2+$ \\
\hline Normal & - & 11 & 35 & 1 & 59 \\
\hline Total & 7 & 15 & +4 & 1 & 65 \\
\hline
\end{tabular}

*Child with ec\%ema.

tChild with upper respiratory infection on study night. sometimes or often fell asleep while reading or watching television, and $27 \cdot 3 \%$ considered their children to be hyperactive. A total of $12 \cdot 1 \%$ snored on most nights and $25 \cdot 7 \%$ snored often, even without a cold. Restless sleep was reported by $31 \cdot 1 \%$ and mouth breathing by $17 \cdot 5 \%$ of parents, whereas $9 \cdot 8 \%$ remembered being told at one time that their child had enlarged tonsils. Nine children had had a tonsillectomy, four of these had by chance been investigated in this department between one and one and a half years earlier and found to have a clear cut sleep and breathing disorder before their tonsillectomy.

\section{Questionnaire interrelations}

Trend analysis $\left(\chi^{2}\right)$ showed the increasing prevalence of daytime sleepiness, hyperactivity, and restless sleep across the four snoring categories to all be significant (table 1). Daytime sleepiness, hyperactivity, and restless sleep were all significantly more common among the habitual snorers (that is, most nights) compared with the never snorers (table 1 ).

SLEEP MONITORING AND BEHAVIOURAL

ASSESSMENT GROUPS

Sleep monitoring results

Overnight video recordings were refused or technically unsatisfactory in $12 / 132$ children (seven from the high risk group), and oximetry recordings were not available for the same reasons in another 15 children (seven from the high risk group). Table 2 summarises the results of home sleep monitoring.

In the high risk group 20/66 (30\%) children had values for nocturnal movement, $>4 \% \mathrm{SaO}_{2}$ dip rate, or overnight pulse rate greater than the 95th centile for normal children. In seven there was clear cut snoring with recurrent upper airway obstruction and consequent sleep disturbance. Another 15 children snored on the night of the study and four of them had abnormal oximetry records, but review of the video recording did not show snoring related sleep disturbance and so they could not be categorised as having a sleep and breathing disorder. One child had eczema with scratching, which explained the high nocturnal movement. Review of the remaining eight children with abnormal oximetry, pulse rate, or movement records showed apparently normal sleep with no snoring or other explanation for their results.

In the control group $6 / 66(9 \%)$ of these children had abnormal overnight monitoring results. This could be explained on the basis of eczema in 
Table 3 Results of overnight sleep monitoring in the high risk and control group (excluding the seven children with an identifiable sleep and breathing disorder)

\begin{tabular}{|c|c|c|c|}
\hline & $\begin{array}{l}\text { Control } \\
\text { group } \\
(n=58)\end{array}$ & $\begin{array}{l}\text { High risk } \\
\text { group } \\
(n=52)\end{array}$ & p Value \\
\hline $\begin{array}{l}\text { \% Time spent moving }(\mathrm{SD}) \\
>4 \% \mathrm{SaO}_{2} \text { dip rate }(\mathrm{SD}) \\
\text { Mean overnight pulse rate }(\mathrm{SD})\end{array}$ & $\begin{array}{r}4 \cdot 9(2 \cdot 3) \\
1 \cdot 4(0 \cdot 8) \\
81 \cdot 0(7 \cdot 2)\end{array}$ & $\begin{array}{r}5 \cdot 8(1 \cdot 9) \\
2 \cdot 1(1 \cdot 4) \\
83 \cdot 8(7 \cdot 2)\end{array}$ & $\begin{array}{l}0.043 \\
0.0006 \\
0.031\end{array}$ \\
\hline
\end{tabular}

one and upper respiratory infection in another two children (which had not been apparent during the interview the previous evening). The other three had apparently normal sleep. Only one child $(1 / 66,2 \%)$ in this group snored on the night of monitoring and her results were within the normal range.

\section{Prevalence of sleep and breathing disorders}

We identified seven children with a detectable sleep and breathing disorder during this study from our original population of 996 children, a prevalence of $0.7 \%$. If we include the four children from this population who had been identified previously (see questionnaire results section) with a sleep and breathing disorder and who are unlikely to have improved without adenotonsillectomy then our estimate of the prevalence of these disorders is even higher at $1 \cdot 1 \%$ $(7+4 / 996)$. This is likely to be an underestimate as we studied only $16 \cdot 8 \%$ of the original population.

\section{Comparison of high risk and control groups}

Initial analysis of the overnight sleep monitoring results showed that the high risk group had significantly higher nocturnal movement, $>4 \%$ $\mathrm{SaO}_{2}$ dip rate, and pulse rate than the controls, but this included the seven children identified with an obvious sleep and breathing disorder (who by definition had high nocturnal movement, $>4 \% \mathrm{SaO}_{2}$ dip rate, and pulse rate). These seven children were therefore subsequently excluded from the comparison of the high risk and control groups. The high risk group nevertheless had significantly higher nocturnal movement, $>4 \% \mathrm{SaO}_{2}$ dip rate, and overnight pulse rate (table 3 ). Thus, even though these children did not have a significant sleep and breathing disorder by our criteria, their separation according to the questionnaire results reflected small but measurable physiological differences in sleep.

Table 4 Relationships between high risk $(n=66)$ and control $(n=66)$ groups and social class and parental smoking

\begin{tabular}{|c|c|c|c|c|}
\hline & \multirow{2}{*}{\multicolumn{2}{|c|}{ Questionnaire ranking }} & \multicolumn{2}{|c|}{ Odds ratio and $95 \%$ confidence interval } \\
\hline & & & \multirow{2}{*}{$\begin{array}{l}\text { Before allowance for } \\
\text { other variables }\end{array}$} & \multirow{2}{*}{$\begin{array}{l}\text { After allowance } \\
\text { for other } \\
\text { variables }\end{array}$} \\
\hline & Control group & High risk group & & \\
\hline \multicolumn{5}{|l|}{ Social class: } \\
\hline Manual (\%) & 36 & 66 & $3 \cdot 26$ & 1.65 \\
\hline \multirow{2}{*}{\multicolumn{5}{|c|}{ Mother: }} \\
\hline & & & & \\
\hline Non-smoker (\%) & 77 & 54 & 2.99 & $4 \cdot 36$ \\
\hline Smoker (\%) & 23 & 46 & $(1.40$ to 6.40$)$ & $(1 \cdot 45$ to $13 \cdot 1)$ \\
\hline \multicolumn{5}{|l|}{ Father: } \\
\hline Non-smoker (\%) & 76 & 64 & $1 \cdot 78$ & 1.41 \\
\hline Smoker (\%) & 24 & 36 & $(0.83$ to 3.28$)$ & $(0.48$ to 2.51$)$ \\
\hline
\end{tabular}

Children in the high risk group were significantly more likely to have a mother who smoked. This was independent of the effect of social class (table 3). Conversely, the increased likelihood of being in the high risk group associated with coming from a manual socioeconomic class was lost after allowing for the effect of maternal smoking (table 4). The increased risk associated with paternal smoking was not significant. There was no difference between the two groups with respect to the prevalence of regular nocturnal enuresis (11/66 in the high risk and 10/66 in the control group).

Eighty six completed parent questionnaires were returned and analysed (34/66 from the high risk group and 52/66 from the control group), and 85 completed teacher questionnaires were returned (35/66 from the high risk and 50/66 from the controls).

Table 5 shows the median and 95th centile scores for each of the subscales. The high risk group had higher scores for all the subscales except the teacher reported inattentive behaviour. These data were transformed as described under Methods for use in logistic modelling.

Social class appeared initially to be a significant predictor of parental Conners scores on univariate analysis. After allowing for the effects of high risk/control category first, however, it no longer remained a significant independent predictor. This was due to a relation between social class and high risk/control category, which in turn was through the maternal smoking effect referred to earlier (table 4). Although social class did not significantly affect the Conners scores independently, it was nevertheless allowed for first in calculating the subsequent odds ratios.

Although the groups overall were matched for gender, this was not so for the subset for which we had Conners results. As boys tend to score higher on the Conners scale $^{9}$ than girls it was allowed for as a possible confounding variable.

Table 6 gives the full logistic modelling results. Children in the high risk group were significantly more likely to be thought inattentive and hyperactive by their parents and teachers. Aggressive behaviour was significantly more likely to be reported by parents of the high risk children. The trend towards increased aggressive behaviour reported in the teacher questionnaire for this group was not significant. Entering social class and gender into the model first did not significantly change the odds ratios for any of the subscales.

\section{Discussion}

This is the first community based study to report the prevalence of sleep and breathing disorders in children. None of the children we identified in this study had severe obstructive sleep apnoea, but a less severe form of obstructive snoring (with some apnoeas) which nevertheless leads to recurrent sleep disruption (evidenced by higher nocturnal movement), greater $>4 \% \mathrm{SaO}_{2}$ dip rate, and higher pulse rate was seen. We have also found significant differences in the results of sleep monitoring and daytime behaviour between children defined as being at high risk of 
Table 5 Conners subscale scores for high risk and control groups as defined by questionnaire ranking; the median and 5th to 95th centiles are shown. The maximum score on any subscale is 3

\begin{tabular}{lll}
\hline Conners subscale & Control group & High risk group \\
\hline Aggressive behaviour (teacher) & $0 \cdot 06(0-0 \cdot 87)$ & $0 \cdot 12(0-0 \cdot 87)$ \\
Aggressive behaviour (parent) & $0 \cdot 33(0-1 \cdot 16)$ & $0 \cdot 81(0-2 \cdot 06)$ \\
Inattentive behaviour (teacher) & $0 \cdot 50(0-1 \cdot 16)$ & $0 \cdot 50(0-2 \cdot 06)$ \\
Inattentive behaviour (parent) & $0 \cdot 33(0-1 \cdot 16)$ & $0 \cdot 75(0-2 \cdot 16)$ \\
Hyperactive behaviour (teacher) & $0 \cdot 06(0-0 \cdot 87)$ & $0 \cdot 13(0-1 \cdot 81)$ \\
Hyperactive behaviour (parent) & $0 \cdot 14(0-0 \cdot 85)$ & $0 \cdot 42(0-2 \cdot 42)$ \\
\hline
\end{tabular}

a sleep and breathing disorder (according to parental history) compared with controls.

\section{QUESTIONNAIRE RESULTS}

Our questionnaire was primarily designed to identify children with sleep and breathing disorders for the second phase of the study. It was derived from a questionnaire used in a previous study of children before a tonsillectomy. ${ }^{7}$ The questions which had proved most predictive of the presence of sleep and breathing disorders were retained as well as a few others about daytime behaviour. The usefulness of the questionnaire results in defining a group at high risk for sleep and breathing disorders is clearly shown by the results of overnight monitoring, which tended to confirm parental history. A response rate of $79 \%$ was achieved after one reminder; we did not pursue this further because our main concern was to find children with sleep and breathing disorders. It is likely that parents who perceived their children as having a sleep problem would be motivated to complete and return the questionnaire. This is supported by the finding that parents were more likely to agree to the sleep study if their child was at high risk of a sleep and breathing disorder $(90 \%$ accepted compared with $75 \%$ of control subjects approached; see under Subjects). There is therefore no reason to believe that there are significant numbers of children with sleep and breathing disorders among the non-responders. A total of $12 \cdot 1 \%$ of children in our study were reported to snore on most nights, a higher figure than that reported by Corbo et al. ${ }^{18}$ The children in our study were considerably younger than their sample and this may explain some of the differ-

Table 6 Distribution of 95th centile scores on Conners subscales according to whether the child was in the high risk or control group. The odds ratio are calculated before and after allowing for gender and social class as possible confounding variables

\begin{tabular}{|c|c|c|c|c|}
\hline \multirow{3}{*}{$\begin{array}{l}\text { Conners subscale } \\
\text { and centile }\end{array}$} & \multirow{2}{*}{\multicolumn{2}{|c|}{ Questionnaire ranking (\%) }} & \multicolumn{2}{|c|}{ Odds ratio and $95 \%$ confidence interval } \\
\hline & & & \multirow{2}{*}{$\begin{array}{l}\text { Before allowance for } \\
\text { other variables }\end{array}$} & \multirow{2}{*}{$\begin{array}{l}\text { After allowance } \\
\text { for other } \\
\text { variables }\end{array}$} \\
\hline & Control group & High risk group & & \\
\hline \multicolumn{5}{|c|}{ Aggressive behaviour (teacher) } \\
\hline $\begin{array}{l}<95 \text { th } \\
>95 \text { th }\end{array}$ & $\begin{array}{l}92 \\
8\end{array}$ & $\begin{array}{l}86 \\
14\end{array}$ & $\stackrel{1.9}{(0.4 \text { to } 7.9)}$ & $\begin{array}{l}1.5 \\
(0.3 \text { to } 6 \cdot 7)\end{array}$ \\
\hline \multicolumn{5}{|c|}{ Aggressive behaviour (parent) } \\
\hline $\begin{array}{l}<95 \text { th } \\
>95 \text { th }\end{array}$ & 94 & $\begin{array}{l}61 \\
39\end{array}$ & $\begin{array}{l}9 \cdot 9 \\
(2 \cdot 4 \text { to } 39 \cdot 4)\end{array}$ & $\stackrel{9 \cdot 3}{(2 \cdot 3 \text { to } 39 \cdot 1)}$ \\
\hline \multicolumn{5}{|c|}{ Inattentive behaviour (teacher) } \\
\hline $\begin{array}{l}<95 \text { th } \\
>95 \text { th }\end{array}$ & $\begin{array}{l}92 \\
8\end{array}$ & $\begin{array}{l}71 \\
29\end{array}$ & $\stackrel{4 \cdot 6}{(1 \cdot 3 \text { to } 21 \cdot 4)}$ & $\stackrel{5 \cdot 6}{(1 \cdot 5 \text { to } 21 \cdot 4)}$ \\
\hline \multicolumn{5}{|c|}{ Inattentive behaviour (parent) } \\
\hline $\begin{array}{l}<95 \text { th } \\
>95 \text { th }\end{array}$ & $\begin{array}{c}94 \\
6\end{array}$ & $\begin{array}{l}73 \\
27\end{array}$ & $\begin{array}{l}5 \cdot 8 \\
(1 \cdot 4 \text { to } 23 \cdot 8)\end{array}$ & $\stackrel{5 \cdot 1}{(1 \cdot 2 \text { to } 21 \cdot 8)}$ \\
\hline \multicolumn{5}{|c|}{ Hyperactive behaviour (teacher) } \\
\hline $\begin{array}{l}<95 \text { th } \\
>95 \text { th }\end{array}$ & $\begin{array}{r}96 \\
4\end{array}$ & $\begin{array}{l}74 \\
26\end{array}$ & $\stackrel{8 \cdot 4}{(1.6 \text { to } 42 \cdot 0)}$ & $\begin{array}{l}7 \cdot 4 \\
(1 \cdot 4 \text { to } 36 \cdot 4)\end{array}$ \\
\hline \multicolumn{5}{|c|}{ Hyperactive behaviour (parent) } \\
\hline $\begin{array}{l}<95 \text { th } \\
>95 \text { th }\end{array}$ & $\begin{array}{c}94 \\
6\end{array}$ & $\begin{array}{l}62 \\
38\end{array}$ & $\stackrel{10 \cdot 1}{(2 \cdot 5 \text { to } 40 \cdot 3)}$ & $\begin{array}{l}9 \cdot 5 \\
(2 \cdot 3 \text { to } 39 \cdot 6)\end{array}$ \\
\hline
\end{tabular}

ence in our results. In their study, reported snoring decreased with age probably because of an increase in pharyngeal dimensions and adenotonsillar atrophy. They excluded children who slept alone from their analysis because snoring was reported considerably less often in these children than in those who shared a room. We did not examine this factor in our study, though we found that parents from lower socioeconomic groups were more likely to have their assessment of snoring confirmed during our overnight monitoring, perhaps because of their more cramped living conditions. If we had also excluded children sleeping alone then our prevalence of reported snoring might have been even higher than $12 \cdot 1 \%$.

Daytime sleepiness, restless sleep, and hyperactive behaviour all showed a significant increasing trend across the snoring categories, hinting at a dose response relation. In adults, snoring is associated with sleep disruption ${ }^{19}$ and self reported daytime sleepiness. ${ }^{20}$ Mild to moderate daytime sleepiness is a subjective symptom and by relying on parental observation we are likely to underestimate the relation. Hyperactive behaviour has been reported in children with sleep and breathing disorders and largely resolves after an adenotonsillectomy. ${ }^{+71}$ Although hyperactivity was subjectively defined in our questionnaire, its strong relation with snoring in this large community sample, confirming other clinical studies, suggests a true association.

We found no difference in the rates of reported or video confirmed snoring between the sexes, similar to the findings of Corbo et al. ${ }^{18}$ This is in stark contrast with adults, where there is a large male preponderance, presumably because the mechanisms of pharyngeal narrowing in children are different from those in adults where neck obesity is a major factor."2

\section{SLEEP STUDY AND BEHAVIOURAL ASSESSMENT}

Selection of children for overnight study

We studied only $132 / 782(16 \cdot 9 \%)$ of the children for whom we had completed questionnaires, and may have therefore underestimated the true prevalence of sleep and breathing disorders because of under reporting by parents. To study all the children in the way we did would have been beyond our capacity. The alternative approach of studying a larger number of children with simpler techniques, such as oximetry alone, would be inappropriate because upper airway obstruction can cause significant sleep disturbance and daytime symptoms without hypoxaemia $^{721}$ and would furthermore not have allowed us to determine the cause of any oximetric abnormalities.

Only one snorer was detected in the control group compared with 15 snorers and seven with sleep and breathing disorders in the high risk group, indicating that our ranking of the children according to the questionnaire responses had a physiological basis.

Comparison of high risk and control groups The significant differences in sleep monitoring 
results between the two questionnaire defined groups indicates that parental responses reflect real and measurable differences in their children's sleep. Nevertheless only one third (22/66) of children in the high risk group actually snored on the night of the study. Croft et al also found parental history poorly predictive of whether a child snored or not during a single night of monitoring. ${ }^{23}$

Are parents simply unreliable witnesses or do their responses represent an average statement about their children's sleep stretching back over a considerable period of time? If the latter is true then one night of sleep monitoring may not provide an adequate description of a child's average sleep. It is possible that children from the high risk group have nasal and pharyngeal dimensions that are critically balanced such that minor upper respiratory infections could lead to upper airway obstruction, snoring, and restless sleep. One of the questions used in ranking the children was the frequency of 'coughs and colds' (question 5, see appendix), and therefore children in the high risk group had more upper respiratory infections than the controls. Our decision not to study children with upper respiratory infections until they had recovered is likely to have underestimated the problems that children in the high risk group experience at night. This may have biased our study against confirming the parental reports of snoring. Despite this possible bias we nevertheless found significant differences in the overnight monitoring of the two groups. The relative weight that should be given to parental reports of a childs' sleep versus a night of monitoring is important in dealing clinically with individual children and in interpreting the results of large scale questionnaire surveys such as this. No studies have yet addressed the issue of night to night or even week to week variability in the results of sleep monitoring in children with mild sleep and breathing disorders. If night to night variability is the explanation for the negative sleep studies in some of our children from the high risk group (with their behaviour differences), then it would be important to measure this variability as clinical decisions are often based on a single night of monitoring. In severe cases one night is probably sufficient.

Children from the high risk group were more likely to come from a manual socioeconomic group, though after allowance for the effects of parental smoking it was no longer significant. Maternal smoking exerted a more powerful effect than paternal smoking, presumably because this reflects more closely the child's passive smoking environment. These findings confirm previous reports which have linked passive smoking, adenotonsillectomy, ${ }^{24}$ and snoring in children. ${ }^{18}$

We shortened the Conners rating scale by using only those questions which make up three of the five subscales normally used. This was to concentrate on those particular behaviour patterns reported to be associated with sleep and breathing disorders in children. The Conners scale is the most widely used research scale in child psychopathology, with good stability over time and inter-rater agreement. ${ }^{25}$ The scale is well validated with significant agreement between the particular subscales and direct observation of children. ${ }^{26}$

There were clear differences using this behaviour rating scale between the two groups, high risk and control, identified by the original questionnaire. This strongly suggests that poorer behaviour in children is associated with disturbed sleep due to upper airway obstruction.

There was a considerable range in the Conners scores of our children, similar to that found in other studies. ${ }^{27}$ The high risk group was concentrated mainly at the higher end of this range, but only about $10 \%$ of their scores actually exceeded the mean levels found in children referred to a south London psychiatric clinic. ${ }^{27}$ Thus they are clearly not as disturbed as this referred group.

It is possible that our results are biased by a tendency for some parents to generally be over critical of their child, thus producing high scores on the original questionnaire and the more formal behavioural assessment. We think this is unlikely for two reasons, however. First, the teachers' behavioural assessments were also different for the two groups, albeit with lower scores than the parents (a phenomenon that has been noted before ${ }^{28}$ ). Second, there were measurable physiological differences during sleep between the two groups showing that the answers to the original questionnaire were not simply reflecting different parental attitudes to their children.

Other potential confounding factors such as social class and gender were allowed for in the analysis and did not alter the results. One possible confounding variable that we have not formally examined is the known relation between behaviour problems and the deafness of secretory otitis media, ${ }^{28}$ which is itself associated with adenotonsillar hypertrophy. This is unlikely to be the explanation for our results for although teachers report more behaviour problems in children with secretory otitis media, no such differences were found for parental reports in the same study. ${ }^{28}$ Furthermore only two children in the high risk group (and none of the controls) had had hearing problems (due to secretory otitis media, one with grommets). Two clinical studies have found an association between daytime sleepiness, behaviour problems, and sleep disturbance due to snoring alone (that is, without hypoxaemia or sleep apnoea). ${ }^{72}$ These two studies showed improvements following adenotonsillectomy, supporting the view that this is a causal relation. In adults, snoring (without apnoea, hypopnoea, or hypoxaemia) can also cause sleep disruption, ${ }^{19}$ and is associated with daytime sleepiness. ${ }^{20}$ It is likely that similar mechanisms operate in children, though they may show other behavioural problems besides sleepiness. Many of the children from the high risk group did not have a significant sleep and breathing disorder on the night of study and yet they displayed considerable behavioural differences from the controls. This suggests either that the small but significant differences in nocturnal movement, desaturation, and pulse rate between the groups reflect biologically important differences in sleep quality, or (if the parental history is to be 
believed) that even intermittent snoring and sleep disruption can have deleterious effects on behaviour.

\section{CONCLUSIONS}

We did not identify any children with severe sleep and breathing disorders in this survey suggesting that parents, general practitioners, and ear, nose, and throat surgeons identify and treat most children with severe problems. Of the children with confirmed snoring or sleep and breathing disorders that we identified, however, more than a third had a history of enlarged tonsils. Presumably in the past, when a more liberal attitude towards tonsillectomy prevailed, these children and perhaps many of the other children in the high risk group would have had an operation. Children with a history of snoring are more likely to be sleepy during the day and to have a number of 'difficult' behaviour patterns than those without such a history. Longitudinal studies, such as the Dunedin survey of otitis media with effusion,,$^{28}$ are required to assess the impact of these nocturnal and behavioural differences on childrens' educational and social development. It will then be possible to determine if current criteria for adenotonsillectomy exclude children who might benefit from having their tonsils removed. Such a study would also help to define what constitutes a 'significant' sleep and breathing disorder in children.

N J Ali is a British Lung Foundation Research Fellow, D J Pitson is supported by Wellcome Trust, and J R Stradling is a Wellcome senior research fellow.

1 Brodsky L, Moore L, Stanievich JF. A comparison of tonsillar size and oropharyngeal dimensions in children with obstrucsize and oropharyngeal dimensions in children with obstruc-
tive adenotonsillar hypertrophy. Int $\mathcal{f}$ Pediatr Otorhinotive adenotonsillar hypertro
laryngol 1987; 13: 149-56.

2 Fry J. Are all 'T's and A's' really necessary? BMF 1957; i: $124-9$.

3 Black N. Geographical variation in use of surgery for glue ear. FR Soc Med 1985; 78: 631-7.

4 Guilleminault C, Korobkin R, Winkle R. A review of 50 children with obstructive sleep apnea syndrome. Lung 1981 159: $275-87$

5 Check WA. Does drop in $T$ and A's pose new issue of adenotonsillar hypertrophy? $\mathcal{F} A M A$ 1982; 247: 1229-30.

6 Rosenfeld RM, Green RP. Tonsillectomy and adenoidectomy: changing trends. Ann Otol Rhinol Laryngol 1990; 99: 18791.

7 Stradling JR, Thomas G, Warley ARH, Williams P, Freeland A. Effect of adenotonsillectomy on nocturnal hypoxaemia A. Efrect of adenotonsillectomy on nocturnal hypoxaemia,
sleep disturbance, and symptoms in snoring children. Lancet sleep disturbance, a

8 Office of Population and Census and Surveys. Classification of occupations. London: HMSO, 1980.

9 Trites RL, Blouin AGA, Laprade K. Factor analysis of the Conners teacher rating scale based on a large normative sample. $\mathcal{F}$ Consult Clin Psychol 1982; 50: 615-23.

10 Vos PJE, Stradling JR. Assessment of sleep times and movement arousals from video recordings. Fournal of Ambulatory Monitoring 1991; 4: 35-42.

11 Warley AR, Mitchell JH, Stradling JR. Evaluation of the Ohmeda 3700 pulse oximeter. Thorax 1987; 42: 892-6.

12 Stradling JR, Crosby JH. Relation between systemic hypertension and sleep hypoxaemia or snoring: analysis in 748 men drawn from general practice. BMF 1990; 300: 75-8.
13 Conners CK. A teacher rating scale for use in drug studies with children. Am f Psychol 1969; 126: 884-8.

14 Cody RP, Smith JK. Applied statistics and the SAS programming language. New York: Elsevier, 1987.

15 Payne C. The GLIM systems release 3.77 manual. Oxford: Numerical Algorithms Group, Royal Statistical Society, 1985.

16 Altman DG. Practical statistics for medical research. London: Chapman and Hall, 1991.

17 Gardner MJ, Altman DG. Statistics with confidence. London: BMA, 1989.

18 Corbo GM, Fuciarelli F, Foresi A, De-Benedetto F. Snoring in children: association with respiratory symptoms and passive smoking [published erratum appears in $B M \mathcal{F} 1990$ 300: 226]. $B M F$ 1989; 299: 1491-4

19 Guilleminault C, Stoohs R, Duncan S. Snoring (I). Daytime sleepiness in regular heavy snorers. Chest 1991; 99: 40-8.

20 Stradling JR, Crosby JH, Payne CD. Self-reported snoring and daytime sleepiness in men aged 35-65 years. Thorax 1991; 46: 807-10.

21 Guilleminault C, Winkle R, Korobkin R, Simmons B. Children and nocturnal snoring: evaluation of the effects of sleep related respiratory resistive load and daytime functioning. Eur $\mathcal{F}$ Pediatr 1982; 139: 165-71.

22 Davies RJ, Stradling JR. The relationship between neck circumference, radiographic pharyngeal anatomy, and the obstructive sleep apnoea syndrome. Eur Respir $\mathcal{F}$ 1990; 3: 509-14.

23 Croft CB, Brockbank MJ, Wright A, Swanston AR. Obstructive sleep apnoea in children undergoing routine tonsillectomy and adenoidectomy. Clin Otolaryngol 1990; 15: 307 14

24 Said G, Zalokar J, Lellouch J, Patois E. Parental smoking related to adenoidectomy and tonsillectomy in children. f Epidemiol Community Health 1978; 32: 97-101.

25 Trites RL, Blouin AGA, Ferguson HB, Lynch GW. The Conners teacher rating scale: an epidemiological inter-rate reliability and follow up investigation. In: Gadow $\mathrm{K}$, Loney J, eds. Psychosocial aspects of drug treatment for hyperactivity. Boulder, Colorado: Westview Press, 1981.

26 Rapoport JL, Benoit $M$. The relation of direct home observations to the clinic evaluation of hyperactive school age boys. f Child Psychol Psychiatry 1975; 16: 141-7.

27 Taylor E, Sandberg S. Hyperactive behaviour in English schoolchildren: a questionnaire survey. $f$ Abnorm Child schoolchildren: a questi
Psychol 1984; 12: 143-56.

28 Chalmers DC, Stewart I, Silva P, Mulvena A. Otitis media with effusion in children. Clin Dev Med 1989; 108.

Appendix

Questionnaire used in the postal survey.

1. Does your child have a problem related to sleeping?

$1=$ Never $2=$ Rarely $3=$ Sometimes $4=$ Often

2. Does he/she have trouble getting to or staying asleep?

1=Never 2=Rarely 3=Sometimes 4=Often

3. Does he/she fall asleep during the day particularly when not active (for example watching TV or reading)?

$1=$ Never 2=Rarely 3=Sometimes 4=Often

4. Is he/she considered to be hyperactive?

$1=$ Never $\quad 2=$ Rarely $3=$ Sometimes $\quad 4=$ Often

5. Does he/she get coughs and colds?

$1=$ Never $2=$ Rarely 3=Sometimes $4=$ Often

6. Does he/she snore at night?

$1=$ Never $\quad 2=$ Rarely $3=$ Sometimes $4=$ Most nights (even without a cold)

7. Is the volume of the snoring?

1=Quiet 2=Moderate 3=Loud 4=Very loud

8. Does he/she sweat at night?

$1=$ Never $\quad 2=$ Rarely $3=$ Sometimes $\quad 4=$ Often

9. Is your child a restless sleeper?

$1=$ Never 2=Rarely 3=Sometimes $4=$ Often

10. Does your child sleep with his/her head tipped back?

$1=$ Never 2=Rarely 3=Sometimes $4=$ Often

11. Does your child tend to breathe through his/her mouth rather than nose?

$1=$ Never $\quad 2=$ Rarely $3=$ Sometimes $4=$ Often

12. Have you ever been told that your child has enlarged tonsils?

$1=$ No $2=$ Yes

13. Has your child had his/her tonsils taken out?

$1=$ No $2=$ Yes 